\title{
EXIT: Estado del arte y cómo lo hacemos
}

\section{EXIT: state of the art. How we do it}

\author{
Ricardo Alarcón G. ${ }^{1,4}$, Hilda Arancibia Z. ${ }^{2,4}$, Andrés Caballero E. ${ }^{3,4}$, \\ Patrick Delucchi W. ${ }^{1,4}$, Camilo Iñiguez L. ${ }^{1,4}$, Juan Patricio Ulloa B. ${ }^{1,4}$
}

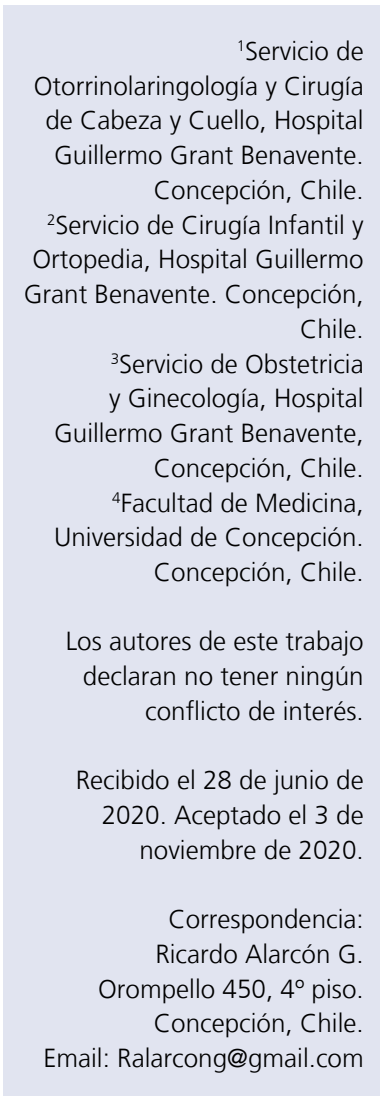

\begin{abstract}
Resumen
El protocolo EXIT (tratamiento ex-útero intraparto) es una técnica que permite establecer una vía aérea segura y estable, en un feto con obstrucción de esta o riesgo al momento del parto. Se basa en mantener la circulación uteroplacentaria con anestesia neonatal, logrando una hipotonía uterina controlada. Todo otorrinolaringólogo que se desempeñe en un hospital que cuente con un servicio de obstetricia de alta complejidad puede ser requerido en este tipo de casos y debe tener un protocolo establecido de cómo proceder. En nuestro medio se trata de un procedimiento poco frecuente que obliga a la revisión de la técnica. No existe un protocolo único en la literatura internacional, pero sí principios fundamentales. Se presenta caso clínico de un embarazo, en que se identifica una masa cervical fetal, realizándose el protocolo EXIT. Se realiza una revisión de la literatura, se describen los principios de esta técnica, así como también nuestro enfrentamiento y lecciones aprendidas.
\end{abstract}

Palabras clave: EXIT, tratamiento ex-útero intraparto.

\begin{abstract}
The EXIT protocol (ex-utero intrapartum treatment) is a technique that allows establishing a safe and stable airway in a fetus with airway obstruction, or at risk of it, at the time of delivery. It is based on maintaining uteroplacental circulation with neonatal anesthesia, achieving controlled uterine hypotonia. Any otolaryngologist working in a hospital that has a highly complex gynecology and obstetric service may be required in this type of case and must have an established protocol of how to proceed. In our setting, it is an infrequent procedure, so it requires a revision of the surgical technique. There is no established protocol in the literature, but there are fundamental principles. We describe a clinical case of a pregnancy where a fetal cervical mass was identified, and an EXIT protocol was performed. A review of the literature is presented, the principles of this technique are described, as well as our procedure and lessons learned.
\end{abstract}

Keywords: EXIT, Ex-utero intrapartum treatment.

\section{Introducción}

Fetos con potencial obstrucción de vía aérea neonatal, como los diagnosticados con masas cervicales congénitas, síndrome de obstrucción de vía aérea alta congénito (CHAOS por sus siglas en inglés) y micrognatia severa requieren de manejo especializado de la vía aérea al momento del parto ${ }^{1}$. Estas patologías han sido asociadas históricamente con pobres resultados, donde la asfixia y muerte perinatal eran frecuentes ${ }^{2-8}$. Esta obstrucción severa de vía aérea alta puede ocasionar graves consecuencias neurológicas por hipoxia o acidosis ${ }^{4}$.

\section{Caso Clínico}

Paciente primigesta, de 25 años, se presenta a una ultrasonografía obstétrica de rutina a las 27 semanas de gestación, donde se pesquisa una masa cervical lateral derecha y anterior a la tráquea, de $30 \times 35 \times 39 \mathrm{~mm}$, con un 
volumen $22 \mathrm{cc}$, sin flujo vascular al doppler, $y$ sin alteraciones en la unidad materno-fetal. El seguimiento ultrasonográfico demuestra crecimiento progresivo y desarrollo de polihidroamnios. Seguimiento con resonancia magnética fetal mostró una lesión quística multilobulada en la región cervical derecha de aproximadamente $66 \times 61 \times 49 \mathrm{~mm}$ en sus ejes mayores, con bordes bien delimitados, sin infiltración grasa ni presencia de calcificaciones, que pudiese corresponder a una malformación vascular linfática (Figura 1). Se evidencia leve efecto de masa sobre estructuras vecinas, sin deformación de la vía aérea. Sin otras malformaciones fetales. Se decide hospitalizar para realizar protocolo EXIT (tratamiento ex-útero intraparto) a las 38 semanas de gestación.

\section{Discusión}

Esta técnica fue originalmente descrita para el manejo de la hernia diafragmática congénita severa $^{2,3}$. El procedimiento está indicado en obstrucciones extrínsecas o intrínsecas de la vía aérea (o riesgo de ellas) que dificulten o impidan la adecuada ventilación posterior al nacimiento, o en que la reanimación neonatal pudiera estar en riesgo, como en masas pulmonares, hipoplasia pulmonar severa, agenesia pulmonar unilateral o tumores de mediastino ${ }^{4}$.

El principal principio del protocolo EXIT es mantener la circulación uteroplacentaria con anestesia neonatal, logrando una hipotonía uterina controlada. Esto permite asegurar la vía aérea fetal, descomprimir o resecar grandes masas cervicales o mediastínicas y servir como puente para proveer acceso vascular para ECMO (oxigenación por membrana extracorpórea), mejorando las posibilidades de supervivencia en estos $\operatorname{casos}^{2-8}$. La identificación antenatal de las lesiones congénitas potencialmente riesgosas de comprometer la vía aérea ha permitido mejorar los resultados permitiendo la coordinación especializada de la asistencia del parto ${ }^{3}$. El adecuado acceso a cuidados prenatales es necesario para su diagnóstico ${ }^{3,6}$. Avances en ultrasonografía y resonancia nuclear magnética han llevado a una mejor delimitación de las lesiones ${ }^{3,5-8}$. Las causas cervicales más frecuentes son los teratomas y malformaciones vasculares linfáticas y venosas $^{3,7}$. Otras masas que pueden presentarse con obstrucción de la vía aérea incluyen quistes tímicos, neuroblastomas, hemangiomas o bocio fetal ${ }^{3}$.

En caso de ser seleccionado para realizarse un EXIT, el embarazo debe permitirse avanzar lo más cercano al término como sea recomendable, idealmente posterior a las 37 semanas, considerando planes de contingencia en caso de que un parto de pretérmino no pueda ser evitado $^{3-5}$. Sin embargo, la mayoría de los casos resulta en polihidroamnios y sobredistensión uterina, debido a la dificultad de la deglución fetal de líquido amniótico, lo que aumenta la posibilidad de un trabajo de parto prematuro. Está reportado hasta un $76 \%$ de trabajo de parto prematuro o rotura de membranas con necesidad de programar EXIT de urgencia. Por
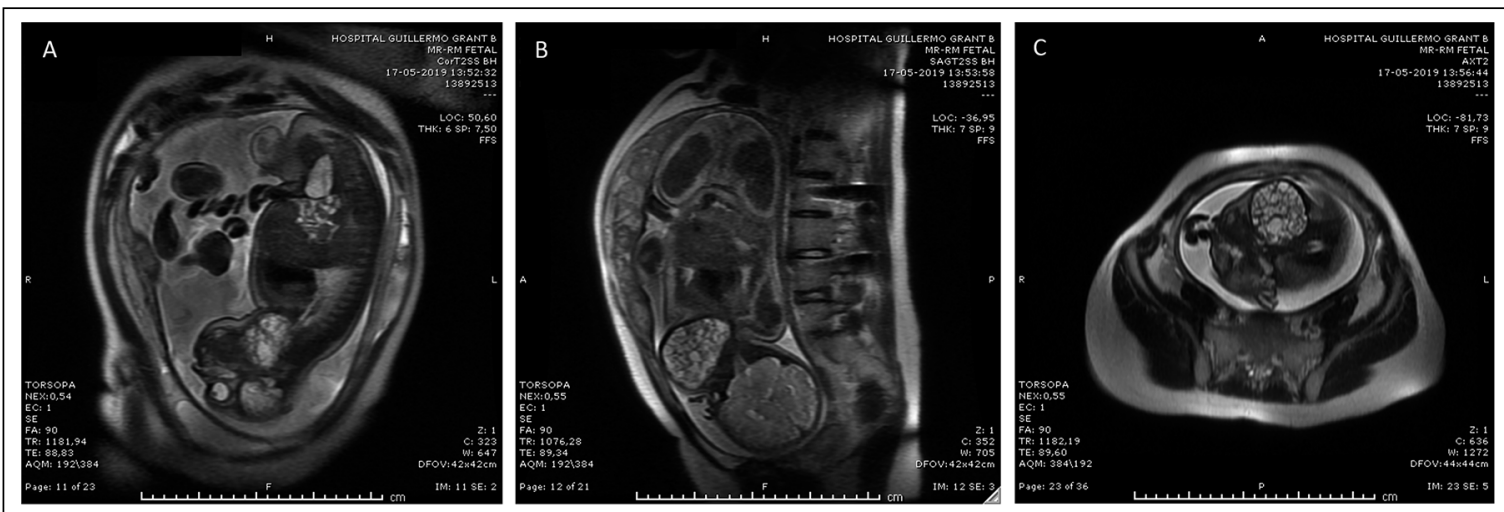

Figura 1. Resonancia magnética que muestra lesión cervical en cortes fetales. (A) RM en corte sagital; (B) RM en corte coronal; (C) RM en corte axial. 
esta razón en promedio los casos reportados de EXIT se realizan alrededor de las 35 semanas de gestación ${ }^{4,8}$.

\section{Enfrentamiento del caso clínico}

Nuestro equipo multidisciplinario incluye 2 otorrinolaringólogos (ORL), arsenalera de otorrino, un tercer otorrinolaringólogo disponible en caso de realizarse traqueostomía (TQT), 2 obstetras y una arsenalera de

\section{Tabla 1. Listado de instrumental EXIT}

1. Laringoscopía

a. Video laringoscopio con rama neonatal y pediátrica y pantalla telepack

b. Aspirador de microcirugía laríngea

c. Tubos endotraqueales números 2,5 - 3- 3,5

d. Pinza Magill pediátrica

e. Óptica cero grados, 2,7 mm

d. Broncoscopio rígido neonatal

f. Laringoscopio de suspensión neonatal

g. Dos cables de luz conectados a fuentes de luz independientes

2 Traqueostomía

a. Caja habitual de traqueostomía con tijeras de iris, separadores Senn-Miller

b. Cánulas traqueostomía 2,5-3-3,5-4

c. Pinza ultrasónica (solo en caso de abordaje transtumoral)

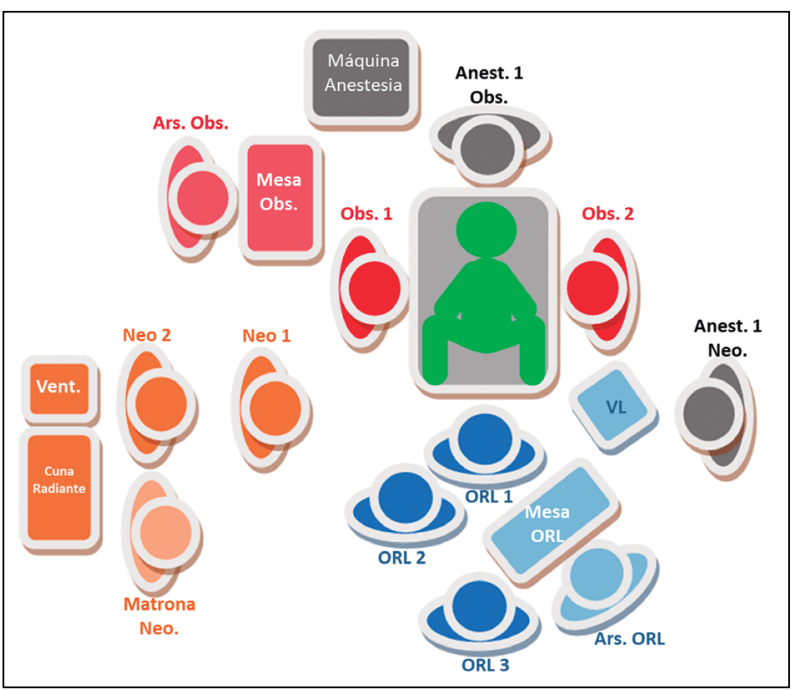

Figura 2. Esquema de la ubicación en el pabellón del equipo multidisciplinario para EXIT. obstetricia, 1 anestesiólogo obstétrico y otro pediátrico, 1 neonatólogo, un cirujano infantil, 1 matrona de neonatología. Estos, entre otros profesionales, son requeridos para orquestar la correcta ejecución de esta técnica.

El enfoque y disposición de los participantes en el pabellón incluye a la embarazada en posición ginecológica sobre mesa quirúrgica al centro del pabellón, máquina de anestesia a la cabeza de la paciente. Obstetra 1 a la derecha de la paciente, obstetra 2 a la izquierda (de pie), mesa de instrumental (Tabla 1) y arsenalera de obstetricia a la derecha de la paciente, por el costado del obstetra 1. ORL 1 entre las piernas de la paciente (sentado) con el monitor del video laringoscopio a su derecha. ORL 2, de pie a la derecha del ORL 1. ORL 3 fuera del campo operatorio (vestido estéril) en espera de ser requerido por una eventual TQT de emergencia. Mesa de instrumental y arsenalera de ORL a la derecha del ORL 1, a los pies de la paciente.

Incubadora con máquina de ventilación mecánica a la derecha de la paciente, equipo de neonatología (neonatólogo, cirujano infantil y matrona) al lado de la incubadora. En caso de requerir una TQT se realizaría con el feto asomado hasta la línea mamaria (para mantener el útero con líquido amniótico y evitar el desprendimiento de placenta), en hiperextensión cervical. En caso de desprendimiento de placenta o esfuerzo respiratorio del feto (ambas situaciones que ponen fin al EXIT por cese de la circulación por el cordón umbilical) la mesa quirúrgica del RN sería la incubadora, equipada con una tabla rígida. Enfermera y pabellonera se mantienen atentas en la entrada del pabellón (Figura 2).

En contraste con la cesárea tradicional, durante el procedimiento EXIT, la tocolisis es el objetivo principal para mantener el soporte placentario (relajación y volumen). La inducción anestésica materna es usualmente realizada con gases halogenados, seguida de una secuencia de intubación rápida y de drogas intravenosas que aseguren las sedación materna e inmovilidad fetal (alternativas son el propofol, fentanilo y/o nitroglicerina $)^{3,5,6}$. Luego de la histerotomía, la cabeza, cuello y un brazo del feto son extraídos del útero, mientras que el cuerpo y cordón umbilical permanecen dentro, para preservación de la oxigenación y temperatura fetal, minimizando la pérdida 
de fluidos ${ }^{3}$. Amnioinfusión con suero Ringer lactato tibio mantiene al feto temperado y el volumen uterino, previniendo la compresión del cordón umbilical y el desprendimiento de placenta ${ }^{3}$. Anestesia intramuscular fetal complementaria es administrada, que incluye sedación, relajante muscular (rocuronio y remifentanyl o ketamina) y atropina (opcionalmente) para evitar el esfuerzo respiratorio y movimientos que impidan realizar el procedimiento. En nuestro caso atropina, fentanil y vecuronio fueron utilizados (Figura 3 ). El monitoreo fetal con oximetría de pulso preductal es necesario ${ }^{3-5}$.

El tiempo efectivo del procedimiento EXIT se inicia una vez que se extrae la cabeza, el cuello y un brazo del feto y concluye ante tres circunstancias:

1. Finalización exitosa del procedimiento (situación ideal).

2. Al primer esfuerzo respiratorio del feto (con lo cual se colapsa el cordón umbilical, interrumpiéndose en el intercambio de oxígeno de la unidad uteroplacentaria) debiendo completarse la cesárea.

3. Desprendimiento de placenta. Circunstancia en la cual, para salvaguardar la vida materna, también debe ser completada la cesárea.

En los dos últimos escenarios se debe asegurar la vía aérea del recién nacido mediante la intubación o la traqueostomía con el paciente en apnea, en la cuna radiante, iniciándose maniobras de reanimación fetal.

Definimos nuestro plan bajo los siguientes pasos:

1. Esperar un minuto antes de cualquier instrumentalización, tras la medicación intramuscular en el deltoides fetal, para que la anestesia y bloqueo intramuscular (del feto) hagan efecto y así disminuir la posibilidad de esfuerzo respiratorio fetal.

2. Intento de intubación nasotraqueal bajo visualización directa con video laringoscopía.

3. Intento de intubación orotraqueal con guía (o broncoscopio).

4. Traqueostomía distal al borde inferior del tumor.

5. De no ser posible lo anterior traqueostomía transtumoral.

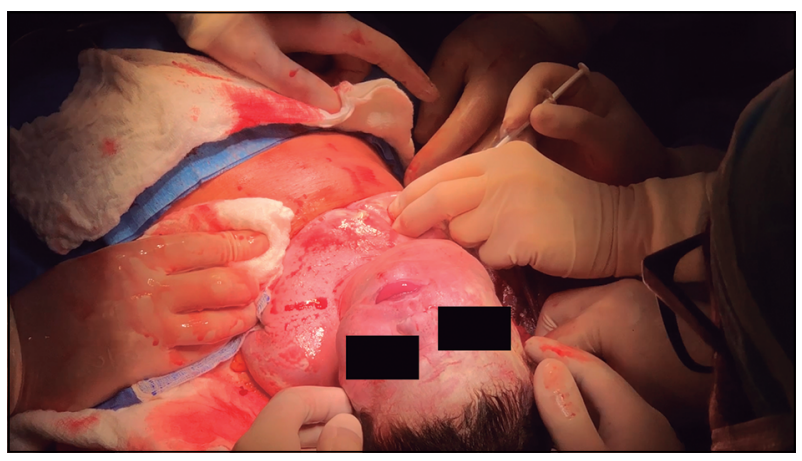

Figura 3. Procedimiento de EXIT.

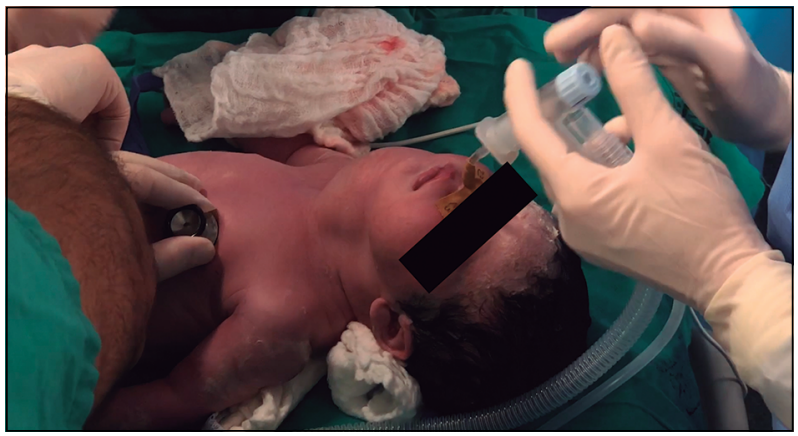

Figura 4. Ventilación de recién nacido en protocolo EXIT.

En nuestro caso fue posible la intubación nasotraqueal sin incidentes. Recomendamos la intubación nasotraqueal para disminuir la posibilidad de desplazamiento mientras se completa el nacimiento o en el traslado a la cuna radiante. El recién nacido luego es ventilado a presión positiva con dispositivo con resucitador manual ${ }^{2}$ (Figura 4 ). Tras asegurar la vía aérea se debe verificar ventilación adecuada, auscultación, capnografía y monitoreo ultrasonográfico de movimiento pulmonar bilateral. Sólo tras verificar una vía aérea segura se procede pinzar y seccionar el cordón umbilical ${ }^{3,4}$.

La utilización de surfactante en forma profiláctica para prevenir barotrauma pulmonar es una opción ${ }^{2}$. Un segundo pabellón debe estar disponible para la completar la resección tumoral si esta fue iniciada durante el EXIT ${ }^{3}$. Siguiendo al parto se suspenden los gases anestésicos y se inician agentes uterotónicos (oxitocina, methergina, ergonovina, misoprostol). Se permite la separación placentaria espontánea y se realiza un cierre por planos anatómicos ${ }^{3,4}$. 
Asegurar la vía aérea es la fase inicial del tratamiento de los neonatos con grandes masas cervicales que pueden comprometer la permeabilidad de la vía aérea. Muchos de ellos seguirán con una vía aérea crítica mientras la masa siga presente. Las opciones de tratamiento dependen del tipo de lesión ${ }^{3}$. Los teratomas, tumores fetales más frecuentes $^{7}$, se benefician de resección en el recién nacido. Estos tienden a desplazar más que a invadir y pueden ser resecados con mínima morbilidad. Si la glándula tiroides está comprometida debe considerarse la posibilidad de hipotiroidismo secundario. Gran esfuerzo se debe hacer para preservar los nervios laríngeos y pares craneanos durante la resección. El seguimiento es necesario para vigilar la recurrencia, evidenciar daño a estructuras nerviosas, deformación mandibular, traqueomalacia o descoordinación orofaríngea ${ }^{3}$.

El manejo de las malformaciones vasculares depende de la característica predominante de la masa. Los hemangiomas y hemangio-endoteliomas pueden ser manejados con escleroterapia, resección quirúrgica o combinación de ambas. Las malformaciones linfáticas y las malformaciones venosas infiltran los tejidos y se extienden entre planos, siendo difíciles de resecar en bloque. Sustancias esclerosantes incluyen doxiciclina, bleomicina, alcohol e hidróxido de potasio, entre otros. Las sustancias esclerosantes causan inflamación en la fase inicial tras su administración, por lo que se deben tomar las precauciones pertinentes para proteger la vía aérea durante la terapia. Múltiples procedimientos pueden ser necesarios y la recurrencia es común ${ }^{3}$.

El pronóstico fetal/neonatal depende de la patología subyacente. La mayoría de las muertes en que la vía aérea ha sido asegurada exitosamente, están asociadas a hipoplasia pulmonar y prematurez ${ }^{3}$. Una traqueostomía o la resección tumoral puede ocasionar sangrado o traumatismo de la vía aérea o estructuras cervicales torácicas adyacentes. Además, se han reportado casos de bradicardia, desprendimiento placentario o compresión del cordón que impiden completar el algoritmo ${ }^{4}$.

El beneficio fetal del EXIT debe ser balanceado con el riesgo materno y su efecto en la fertilidad a largo plazo. El procedimiento puede ser realizado con mínima morbilidad, pero el sangrado sigue siendo una preocupación importante. Diferencias significativas con respecto a la cesárea tradicional han sido encontradas en las pérdidas hemáticas durante el procedimiento (1.104 vs $883 \mathrm{~mL}$ ), infección de herida operatoria (15 vs 2\%). Estudios sobre cirugía fetal intrauterina no muestran diferencias significativas en el desarrollo de endometritis, ni en fertilidad, si bien hubo un 14\% de dehiscencia uterina y ruptura en embarazos subsecuentes. Se presume mejores resultados en caso del EXIT, en que el procedimiento es realizado al final del embarazo y en un segmento uterino más bajo $^{3}$. En una serie de 45 casos, donde 35,6\% fueron de emergencia, fue reportada una pérdida hemática media de $800 \mathrm{~mL}$ (5002.000 ), en las que un $13,3 \%$ de las pacientes recibieron transfusión. La estadía hospitalaria fue de unos 4 días (3-7) 1 .

\section{Conclusión}

El acceso a diagnóstico antenatal es fundamental para la detección de embarazos complicados con obstrucción de la vía aérea fetal. Estos binomios materno-fetales requieren de un seguimiento estricto en centros preparados para controlar y resolver la patología causal del problema y sus posibles complicaciones.

El ensayo, la práctica y simulación del procedimiento permite el intercambio de información y puntos de vista de los participantes y permite prever y anticiparse a requerimientos y necesidades del intra $y$ posoperatorio, junto con establecer una comunicación efectiva en el equipo humano, que en estas instancias es fundamental para el éxito del procedimiento.

El protocolo EXIT es la indicación de elección para el manejo de pacientes con lesiones obstructivas congénitas de la vía aérea. Habilidades múltiples son necesarias para llevarlo a cabo y un equipo multidisciplinario de especialistas entrenados y coordinados es necesario para evitar errores inherentes de un ambiente con una multitud de agentes participantes. La vía aérea debe ser manejada por un individuo que posea las distintas habilidades detalladas previamente, capaz de asegurarla por el método que sea necesario. Es de suma 
importancia que los otorrinolaringólogos de hospitales con maternidades de alta complejidad conozcan el procedimiento y definan su propio plan de acción.

\section{Bibliografía}

1. Shamshirsaz AA, Aalipour S, Erfani H, et al. Obstetric outcomes of ex-utero intrapartum treatment (EXIT). Prenatal Diagnosis. 2019;39(8):643-646.

2. Pucher B, Szydlowski J, Jonczyk-Potoczna K, Sroczynski J. The EXIT (ex-utero intrapartum treatment) procedure - from the paediatric ENT perspective. Acta Otorhinolaryngol Ital. 2018;38(5):480-484.

3. Olutoye OO, Olutoye OA. EXIT procedure for fetal neck masses. Curr Opin Pediatr. 2012;24(3):386-393.

4. Cruz-Martínez R, Méndez A, Pineda-Alemán H.
Técnica EXIT (Ex Útero Intrapartum Treatment): indicaciones, limitaciones, riesgos y evolución a la técnica de intubación traqueal fetal endoscópica (FETI). Ginecol Obstet Mex. 2015;83:58-65.

5. Fernández R, González R, Valdés R. EXIT

(Extrauterine Intrapartum Treatment), primer caso en hospital público chileno. Rev Otorrinolaringol Cir Cabeza Cuello. 2009:69(1):45-50.

6. Daszenies C, Niklitschek E. Técnica EXIT en Chile. Rev Otorrinolaringol Cir Cabeza Cuello. 2015;75:301303.

7. Guerra F, Rodríguez P, Rubilar M, et al. Linfangioma cervical cavernoso en un gemelo: análisis crítico del diagnóstico y manejo perinatal. Rev Chil Obstet Ginecol. 2007; 72(5):334-341.

8. Jiang S, Yang C, Bent J, et al. Ex utero intrapartum treatment (EXIT) for fetal neck masses: A tertiary center experience and literature review. Int J Pediatr Otorhinolaryngol. 2019;127:109642. doi: 10.1016/j. ijporl.2019.109642. 